\title{
卤过氧化物酶在绿色卤化反应中的研究进展
}

\author{
曾志刚 ${ }^{a, b}$ 桑贤轲 ${ }^{a}$ 袁 波 ${ }^{c}$ 吴鸣虎*, $a, b$ 张武元 ${ }^{*, c}$ \\ ( ${ }^{a}$ 湖北科技学院核技术与化学生物学院 湖北咸宁 437100) \\ ( ${ }^{b}$ 辐射化学与功能材料湖北省重点实验室 湖北咸宁 437100) \\ ( ${ }^{c}$ 西安交通大学化学工程与技术学院 西安 710049)
}

\begin{abstract}
摘要 卤化反应是一类极其重要的有机合成反应，在实验室研究和化工生产领域占据重要地位. 传统卤化反应因存在 使用有毒有害试剂、反应缺乏选择性等问题而亟待改进, 生物酶催化策略则为突破上述瓶颈提供了可能. 自然界已经 进化出多种可对有机物中催化引入卤素的卤化酶. 酶催化卤化反应的突出优势在于常温常压下, 可使用来源温和的卤 素进行高效的催化反应. 催化范围包括卤化、羟卤化、卤环合和氧化脱羧等多种具有挑战性的反应. 鉴于酶催化卤化 反应展示出巨大的潜力, 从催化活性、酶稳定性、底物浓度、催化范围等几个方面着重介绍了卤过氧化物酶在绿色卤 化反应中的最新研究进展，为进一步开发绿色的卤化酶催化卤化反应提供参考.
\end{abstract}

关键词 卤过氧化物酶; 生物催化; 卤化反应; 有机合成; 绿色化学

\section{Advances of Haloperoxidases-Catalyzed Green Halogenation Reactions}

\author{
Zeng, Zhigang ${ }^{a, b} \quad$ Sang, Xianke ${ }^{a} \quad{\text { Yuan, } \mathrm{Bo}^{c} \quad \mathrm{Wu}, \text { Minghu }^{*, a, b} \quad \text { Zhang, Wuyuan }}^{*, c}$ \\ ( ${ }^{a}$ School of Nuclear Technology and Chemistry \& Biology, Hubei University of Science and Technology, \\ Xianning, Hubei 437100) \\ ( ${ }^{b}$ Hubei Key Laboratory of Radiation Chemistry and Functional Materials, Xianning, Hubei 437100) \\ ( ${ }^{c}$ School of Chemical Engineering and Technology, Xi'an Jiaotong University, Xi'an 710049)
}

\begin{abstract}
Halogenation reaction is one of the key reactions in organic synthesis, which acts pivotal part both in laboratory study and industrial applications. Traditional halogenation reactions rely on the use of toxic and hazardous reagents with low selectivity. The enzymatic catalysis bears enormous possibilities to overcome abovementioned bottlenecks remaining in traditional halogenation reactions. Nature has evolved a variety of halogenases that are capable of catalyzing the introduction of halogen atoms in organic substrates. The particular advantage of enzymatic catalysis is that it enables the efficient halogenation reactions occurring at common temperature and pressure with the use of mild halogen sources. The enabled challenging reactions range from halogenation, halohydroxylation, halocyclization to oxidative decarboxylation. In view of the significant potential of enzymatic catalysis in halogenation reactions, the recent advances of halogenase-catalyzed green synthesis are introduced from the perspective of catalytic activity, enzyme stability, substrate concentration, catalytic scope and so on. It is hoped to raise further inspirations in the development of green halogenation reactions catalyzed by halogenases.
\end{abstract}

Keywords haloperoxidases; biocatalysis; halogenation reactions; organic synthesis; green chemistry

有机卤素化合物广泛存在于药物和农用化学品中, 并作为合成中间体应用于化学工业的所有领域. 以医药 领域为例, 2019 年全球销售排名前 200 品牌药物中含氟 药物达 30 余种, 年销售额 636.38 亿美元，占总销售额 $14.2 \%{ }^{[1]}$. 卤代药物及其他卤代化合物的需求正在快速
增长. 而作为合成化学中间体时, 因碳-卤键 $(\mathrm{C}-\mathrm{X})$ 可 以更容易地被金属化，卤代化合物在过渡金属催化的交 叉偶联反应中极具价值.

许多传统化学法, 特别是用于芳香族化合物的卤化 方法, 通常需要使用有毒且对环境有害的亲电子卤素

\footnotetext{
* Corresponding authors. E-mail: w.zhang@xjtu.edu.cn; minghuwu@163.com

Received September 2, 2020; revised September 26, 2020; published online October 13, 2020.

Project supported by the National Natural Science Foundation of China (No. 31601675), the Youth Top Talent Program and Tracking Support Program of Xi'an Jiaotong University, and the Ph.D. Start-up Fund of Hubei University of Science and Technology (No. BK201821).

国家自然科学基金(No. 31601675)、西安交通大学青年拔尖人才计划及青年跟踪计划和湖北科技学院博士启动基金(No. BK201821)资助项目.
} 
源. 此类方法选择性较差, 且通常得到卤化区域异构体 的混合产物. 这不仅导致原料利用率低, 而且副产物对 环境有持续污染性, 后续难以处理 ${ }^{[2,3]}$. 基于此, 科研工 作者开展了大量的研究工作, 并取得了积极进展 ${ }^{[4,5]}$. 但 上述研究通常是利用底物的某些碳-氢键 $(\mathrm{C}-\mathrm{H})$ 的细微 酸度差别或需使用导向基团赋予卤化反应的选择 性 ${ }^{[6-11]}$, 导致在底物范围、化学特异性以及反应适用性 等方面受到一定限制. 因此, 开发可靠、简便、清洁的 选择性卤化方法对环境友好型工业的发展至关重要, 契 合当前国家对绿色发展的战略需求. 通往该目标的一个 潜在途径就是利用卤化酶作为生物催化剂 ${ }^{[12-13]}$. 因此, 本文将针对近些年报道的最具合成潜力的卤化酶, 尤其 是卤过氧化物酶在有机合成领域的应用展开讨论, 主要 从酶催化绿色卤化反应的合成实用性角度(催化活性、 稳定性、底物浓度、催化范围等)展开分析评述, 为促进 卤代反应中使用卤化酶作为催化工具提供参考.

\section{1 自然界的卤化酶}

卤化反应，即将化合物结构中的 $\mathrm{C}-\mathrm{H}, \mathrm{C}-\mathrm{O}$ 等化 学键转化为 $\mathrm{C}-\mathrm{X}$ 键以得到相应的卤代化合物. 自然界 中存在多种高效的卤化酶, 它们可以在常温常压下, 利 用温和卤素原料(如氯化钠)高选择性地催化卤化各种生 物合成前体 ${ }^{[12-13]}$. 卤化酶可将特定的惰性 $\mathrm{C}-\mathrm{H}$ 键转化 为 $\mathrm{C}-\mathrm{X}$ 键, 在手性合成、重要化合物砌块及药物合成 修饰等方面均具有重要应用价值. 与许多合成方法一 样, 大自然在很大程度上启发了有机卤素化合物的开发 和应用. 目前, 从微生物分离出的大量卤代天然产物显 示了强大的抗微生物和抗肿瘤活性 ${ }^{[14]}$. 而通过对有机 卤素化合物的生物合成途径研究, 已发掘出多种能够卤 化游离的或被蛋白质束缚的脂类和芳香族底物的酶 类 ${ }^{[15-16]}$.

其中, 知名的有卤代醇脱卤酶(Halohydrin dehalogenases, E.C. 4.5.1. $)^{[17]}$ 、卤化单加氧酶(Halogenases, E.C. 1.14.19. ${ }^{[16]}$ 和卤过氧化物酶 (Haloperoxidases, E.C. 1.11.1. $)^{[18]}$. 从合成化学的角度看, 使用前两类酶都存在 一定问题. 卤代醇脱卤酶依赖环氧化物为底物, 而热力 学平衡不利于开环反应 (即导致可逆反应) ${ }^{[17]}$. 卤化单加 氧酶多用于催化转化芳烃的 $\mathrm{C}-\mathrm{H}$ 键, 具有很高的区域 选择性, 但这类酶因活性低通常需要使用高负载量, 使 其在化学合成的实际应用价值大幅降低 ${ }^{[19]}$. 相反, 卤过 氧化物酶在可靠性和催化活性方面表现优异, 是卤化化 学领域非常有潜力的催化剂. 因此, 下文将重点就卤过 氧化物酶催化温和及低废卤化反应方面的应用进展进 行分析与讨论.

\section{2 卤过氧化物酶的分类及催化机理}

卤过氧化物酶按其能氧化的最具负电性的卤离子 分类. 如氯过氧化物酶 (CPO, E.C. 1.11.1.10) 可以氧化 $\mathrm{Cl}^{-} 、 \mathrm{Br}^{-}$和 $\mathrm{I}^{-}$，溴过氧化物酶(BPO, E.C. 1.11.1.18) 可以 氧化 $\mathrm{Br}^{-}$和 $\mathrm{I}^{-}$, 但不能氧化 $\mathrm{Cl}^{-}$. 虽然早在 2002 年已有 关于氟化酶的报道 ${ }^{[20-21]}$, 但由于 $\mathrm{F}^{-}$的高电负性和氧化 电位，迄今尚未发现可以单纯利用 $\mathrm{H}_{2} \mathrm{O}_{2}$ 来活化 $\mathrm{F}^{-}$的氟 过氧化物酶. 目前研究集中于两类卤过氧化物酶: 血红 素依赖型卤过氧化物酶 ${ }^{[22]}$ 和钒依赖型卤过氧化物酶 (VCPO 或 VBPO $)^{[18]}$. 二者均利用 $\mathrm{H}_{2} \mathrm{O}_{2}$ 生成次卤酸盐 $\left(\mathrm{XO}^{-}, \mathrm{X}=\mathrm{Cl}, \mathrm{Br}, \mathrm{I}\right)$, 以化学剂量的方式催化卤化反应. 但这两类酶在辅基和催化机理上存在显著差异. 血红素 依赖型卤过氧化物酶利用静息状态的血红素和 $\mathrm{H}_{2} \mathrm{O}_{2}$ 形 成具有催化活性的 $\mathrm{Fe}^{4+}=\mathrm{O}$ 复合物(Compound I), 该复 合物氧化卤化物生成对应的活性 $\mathrm{XO}^{-}$(图 1a); 而钒依赖 型卤过氧化物酶利用静息状态的钒离子活性中心与 $\mathrm{H}_{2} \mathrm{O}_{2}$ 侧向结合形成钒过氧中间体, 卤化物对该过氧中 间体的极化氧原子进行亲核进攻，开环并形成 $\mathrm{XO}^{-}$(图 $1 b)^{[23]}$. 两类酶产生的 $\mathrm{XO}^{-}$起化学计量试剂的作用, 在 接下来的反应中被引入到底物中. $\mathrm{XO}^{-}$及单质卤素是有 机合成中常见的氧化剂. 其用途包括醇的氧化、活化烯 烃的卤化及人名反应(Hunsdiecker 反应、Haloform 反应、 Hofmann 重排反应)等. 然而, 由于 $\mathrm{XO}^{-}$的不稳定性、 较高的反应活性(导致安全问题)以及反应中化学计量的 盐废物生成(导致环境问题), 在直接使用 $\mathrm{XO}^{-}$催化卤化 反应时具有一定困难. 另一方面，市售活性次氯酸盐的 有效氯含量偏低且成分复杂，直接添加使用会导致较多 副反应发生. 因此，从绿色和高选择性合成化学角度来 看，使用酶催化是克服上述诸多困难的一个关键途径.

值得一提的是，两类卤过氧化物酶所需的协同底物 (co-substrate) $\mathrm{H}_{2} \mathrm{O}_{2}$ 作为一种绿色氧化剂, 在有机合成领 域倍受青睐 ${ }^{[24]}$. 且基于 $\mathrm{O}_{2}$ 还原制备 $\mathrm{H}_{2} \mathrm{O}_{2}$ 是目前的研究 热点(以替换当前仍在使用的由法本公司于 1940 年左右 开发的蒽醌技术 $)^{[25]}$.

野生的血红素卤过氧化物酶数量有限. 来自于烟熏 细单胞菌(Leptoxyphium fumago, LfCPO, E.C. 1.11.1.10) 的酶最早于 1960 年代被发现，现已被广泛用于氧官能 化反应 ${ }^{[26]}$. 最近, 有研究报道了来自茶树菇 (Agrocybe aegerita, AaeUPO, E.C. 1.11.2)的卤过氧化物酶 ${ }^{[27]}$. Aae$\mathrm{UPO}$ 因其对 $\mathrm{C}-\mathrm{H}$ 键出色地选择性活化及氧化能力, 更 多是作为一种选择性的氧官能化催化剂, 而非卤化催化 剂. 血红素型卤过氧化物酶的催化活性虽然极其出色 (转化频率 $\mathrm{TOF}=10 \sim 350 \mathrm{~s}^{-1}$ ), 但由于对 $\mathrm{H}_{2} \mathrm{O}_{2}$ 的低耐受 

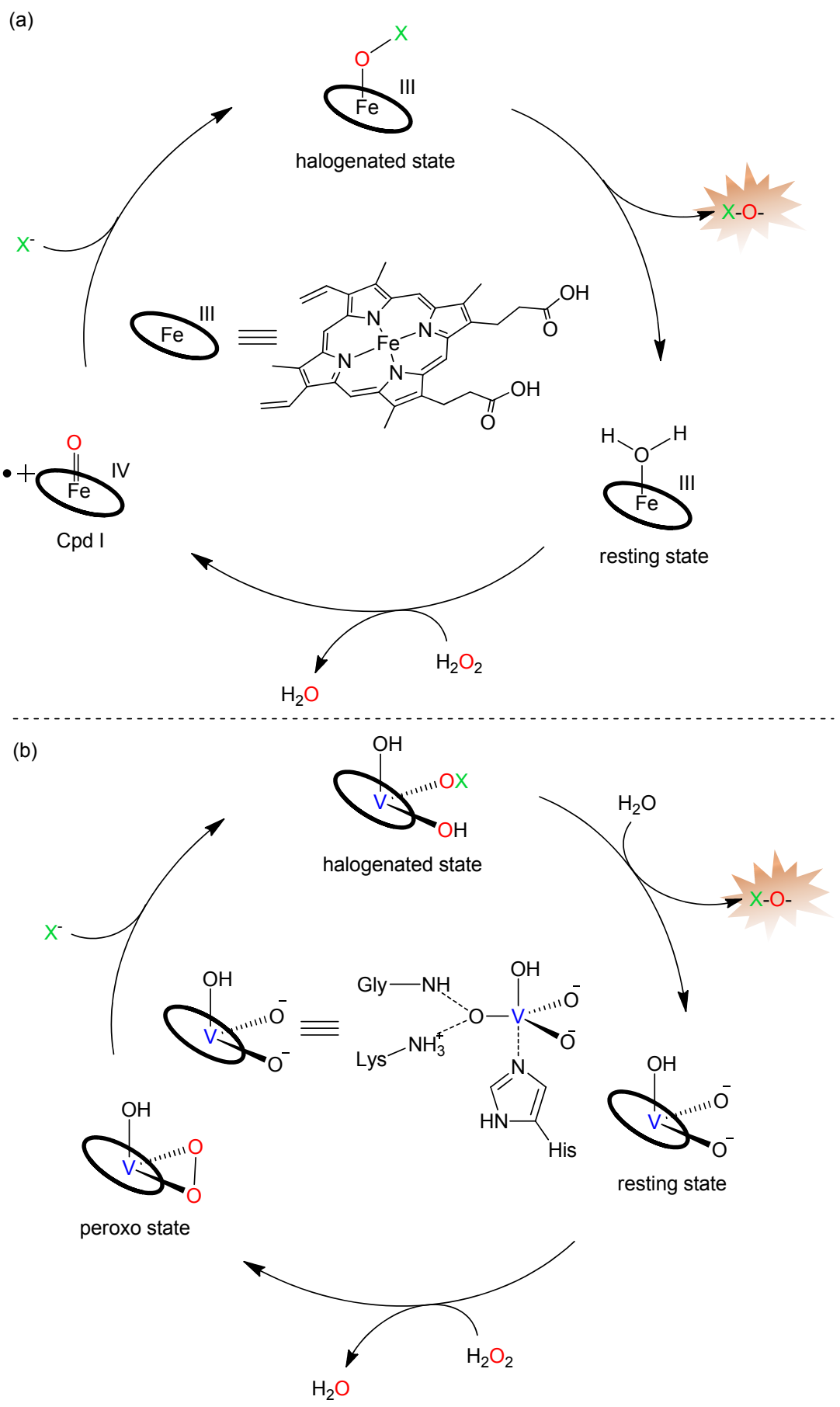

图 1 血红素依赖型(a)和钒依赖型(b)卤过氧化物酶氧化卤素的机理

Figure 1 mechanism of Heme- and vanadium-dependent haloperoxidases catalyzed oxidation of halogens

性使其实用性受阻. 原则上, 可以通过缓慢加料或 $\mathrm{H}_{2} \mathrm{O}_{2}$ 原位生成技术来克服此问题.

相较而言, 主要来自海洋生物的钒依赖卤过氧化物 酶种类丰富, 并且可以从地衣和陆地真菌等其他来源获 得. Wever等 ${ }^{[28-29]}$ 分别于 2013 年和 2019 年对该类酶的微 生物学来源和生物化学机制进行了详细的评述. 相比血 红素型酶, 钒依赖型卤过氧化物酶对 $\mathrm{H}_{2} \mathrm{O}_{2}$ 具有良好的 耐受性, 是其在放大实验和清洁卤化反应中具有前景的
一个重要原因. 其中, 来自不等弯孢菌属的氯过氧化物 酶(Curvularia inaequalis, CiVCPO)表现优异, 在与 100 $\mathrm{mmol} / \mathrm{L}$ 的 $\mathrm{H}_{2} \mathrm{O}_{2}$ 共同储存数天后, 酶的催化活性无明显 降低; 且在高温(最高 $70{ }^{\circ} \mathrm{C}$ ) 和有机助溶剂(乙醇、乙酸 乙酯、丙酮)存在下, 该酶依然保持良好稳定性 ${ }^{[30]}$.

\section{3 卤过氧化物酶的催化反应范围}

作为极具应用潜力的酶, 卤过氧化物酶已被应用于 
多种卤化和氧化反应, 包括富电子芳烃的卤化、 $\mathrm{C}=\mathrm{C}$ 键的卤化羟基化、杂原子氧化和氧化脱羧等. 在接下来 的讨论中, 将对这几大类催化反应中酶的催化性能展开 分析与讨论.

\section{1 氧化反应}

氧化反应是卤过氧化物酶最早的合成应用之一. 张 卫等 ${ }^{[31]}$ 早在 2007 年利用来自珊瑚藻的溴过氧化物酶 (VBPO)催化氧化环己烯制备环氧环己烷, 得到了较出 色的收率与催化选择性, 实现了温和条件下 $\mathrm{KBr}$ 循环利 用，但同时观察到了溴代环己烷等副产物. 2010 年，他 们 $^{[32]}$ 进一步优化了反应条件, 使环氧环己烷的时空收 率达到 $4.79 \mathrm{~g} /(\mathrm{h} \cdot \mathrm{L})$, 同时 $\mathrm{H}_{2} \mathrm{O}_{2}$ 利用效率提高到 $74 \%$. 由 此可见，对反应条件的选择及优化对卤化酶的催化性能 有重要影响. 该研究对以环氧环已烷为底物的有机合成 及高分子合成具有重要意义.

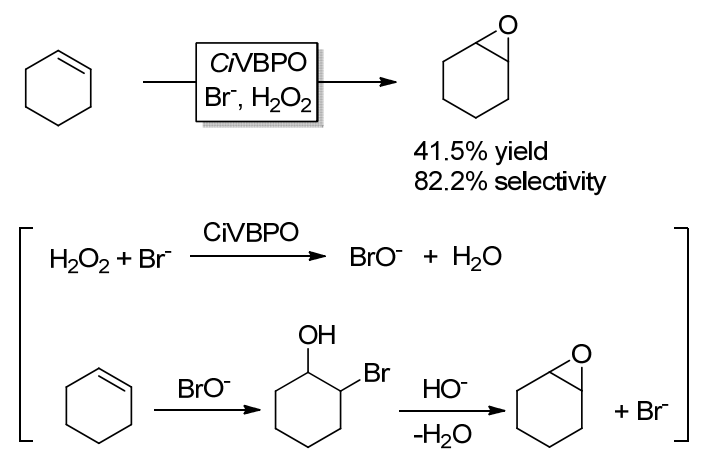

图 2 VBPO 催化环己烯的环氧化反应及反应机理

Figure 2 VBPO catalyzed epoxidation of cyclohexene and the reaction mechanism

\section{2 卤化反应}

卤化反应是另一类研究较多的反应. 由于反应介质 中存在可扩散的次卤酸盐, 因此反应的选择性取决于底 物的化学反应性. 2012 年, Hartung 等 ${ }^{[33]}$ 利用溴过氧化物 酶(VBPO)进行酚的溴化反应时发现, 富电子芳环的取 代基可显著影响卤化反应速率. 酶催化产物为单溴取代 物, 而化学法使用 $\mathrm{Br}_{2}$ 得到三溴取代产物. 因此, VBPO 疑似对酚类物质具有区域选择性, 但尚无令人信服的分 子机理解释. Holtmann 等 ${ }^{[34]}$ 探索了利用血红素依赖型 $L f \mathrm{CPO}$ 来卤化萜烯, 以提高其抗菌活性. 尽管 $L f C P O$ 的 催化活性转换次数(turnover number, TON)高达 160000 , 但底物最高浓度不超过 $2.5 \mathrm{mmol} \cdot \mathrm{L}^{-1}$, 严重限制了在合 成化学中的应用潜力. 2015 年, Hollmann 等 ${ }^{[30]}$ 使用氯过 氧化物酶 $(C i \mathrm{VCPO})$ 溴化百里香酚时发现, 溴化选择性 是动力学控制的. 当初级单溴代产物浓度达到与起始原 料相当的浓度时, 被进一步转化为二溴化产物. 因此, 原则上可通过控制反应时间选择性地得到理想的产物.
除了超高的催化活性(TON $>2000000), C i \mathrm{VCPO}$ 被证明 是非常出色的催化溴代反应的酶(相对于 VBPO). 目前, 该酶已被应用于催化一系列富电子芳香化合物的卤化 反应，展示了广泛的底物范围和催化活性(图 3). 以百里 香酚的卤化反应为例，溴代及氯代的收率在 $80 \%$ 以上. 卤代后的百里香酚的抑菌效果比未卤代的百里香酚有 显著提高 ${ }^{[35]}$, 因此, 该研究在药物的清洁、简便性合成 与修饰方面表现出巨大的应用潜力. 值得一提的是，该 酶可催化转化已经含有卤素的底物，从而可合成不同卤 素取代的目标化合物.

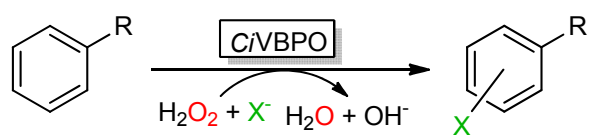<smiles>Cc1cc(O)c(C(C)C)c(Cl)c1</smiles>

$90 \%$<smiles>Cc1cc(O)c(C(C)C)c(Br)c1</smiles>

$86 \%, 81 \%$<smiles>Cc1c(O)cccc1Br</smiles>
$86 \%, 59 \%$<smiles>CC(C)(C)c1c(O)cccc1Br</smiles>

$53 \%, 22 \%$<smiles>[X]c1cc(OC)cc(OC)c1</smiles>

$43 \%, 6 \% \quad 51 \%, 10 \% \quad 90 \%, 62 \% \quad 22 \% \quad 93 \%, 92 \%$<smiles>COC(=O)c1cc(Br)c(Br)[nH]1</smiles><smiles>N#Cc1ccc(Br)c2c1NC(O)(O)C2</smiles>

$40 \%$<smiles>Cc1[nH]c2ccccc2c1Br</smiles><smiles></smiles>

$11 \%$<smiles>COc1ccc(Br)c(OC)n1</smiles>

$34 \%$

图 3 卤过氧化物酶催化合成卤代芳烃的实例及反应的转化 率(蓝色)和收率(绿色) $)^{[30,36-37]}$

Figure 3 Synthesis of halogenated arenes catalyzed by haloperoxidases and the corresponding conversion (blue) and yield (green)

\section{3 羟卤化反应}

另一个有意义的应用是使用卤过氧化物酶将卤素 引入到 $\mathrm{C}=\mathrm{C}$ 双键合成卤代醇. 卤代醇因同时含有两个 官能团，是非常实用的有机合成中间体，在天然产物合 
成方面具有重要应用. 由于酶催化反应在水溶液中进 行, $\mathrm{OH}^{-}$作为亲核试剂, 因此主要产物为卤代醇, 反应 遵循 Markovnikov 规则. 在一些羟卤化反应中, 观察到 了乙烯基卤化物或二醇副产物. 该现象主要是由对卤鎓 离子中间体的氧化脱羒(见下文)或环氧中间体的水解引 起的.

来自小珊瑚藻的 VBPO 和 $L f C P O$ 在较早前已被应 用于羟卤化多种烯烃制备卤代醇, 但量级合成潜力尚未 开发 ${ }^{[38]} .2017$ 年, Hollmann 等 ${ }^{[39]}$ 研究表明, CiVCPO 催化 卤代醇合成具有出色的催化性能: 当催化对苯乙烯磺酸 钠的羟卤化反应 $6 \mathrm{~h}$ 时, 反应达到了全转化, TON 高达 1200000 , TOF $69 \mathrm{~s}^{-1}$ (图 4). 同时, 该酶对芳烯烃、环己 烯和油酸等多类底物均具有出色的催化性能(图 5). 但 在苯乙烯羟卤化反应的量级制备 $(1.1 \mathrm{~g})$ 时, 只获得了 $50 \%$ 的收率, 主要原因在于随着反应的进行, 体系 $\mathrm{pH}$ 逐 渐升高, 引起了产物的环合反应(获得了 $30 \%$ 的环氧苯 乙烯). 因此, 在量级合成时, 对反应体系 $\mathrm{pH}$ 的控制是 一个关键因素.

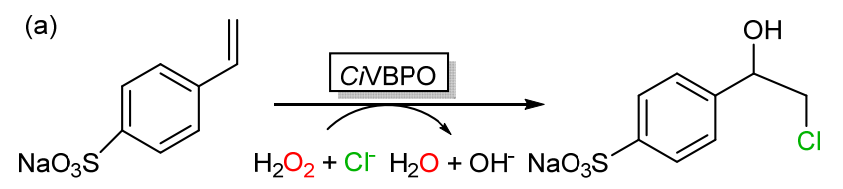

(b)

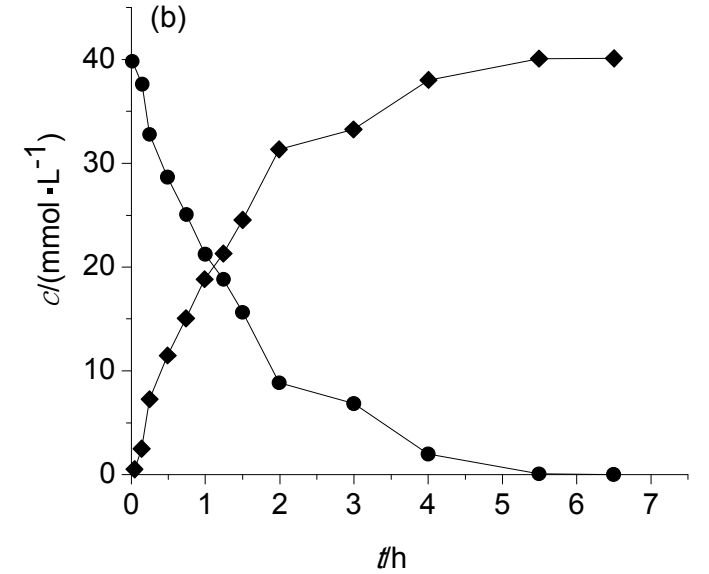

图 $4 \mathrm{VCPO}$ 催化苯乙烯磺酸钠的着直化反应的时间历程(底

物○; 产物

Figure 4 Time course of halohydroxylation of sodium styrene sulfonate catalyzed by VCPO (substrate ; product )

Reaction conditions: aqueous $\left(\mathrm{D}_{2} \mathrm{O}, 0.1 \mathrm{~mol} \cdot \mathrm{L}^{-1}\right.$ citrate buffer, $\left.\mathrm{pH} 5.0\right)$, [sodium styrene sulfonate] $=40 \mathrm{mmol} \cdot \mathrm{L}^{-1},[\mathrm{KBr}]=160 \mathrm{mmol} \cdot \mathrm{L}^{-1}$, $[\mathrm{CiVCPO}]=100 \mathrm{nmol} \cdot \mathrm{L}^{-1}, T=25{ }^{\circ} \mathrm{C}$. The concentration of the substrate and product was determined by ${ }^{1} \mathrm{H}$ NMR

\section{4 卤环合反应}

以 $\gamma, \delta$-不饱和羧酸作为底物时, 卤过氧化物催化卤 环合反应生, 经分子内酯化作用成卤代内酯 ${ }^{[40]}$. 而当底 物为 $\gamma, \delta$-不饱和醇时, 反应经分子内醚化作用过程生成

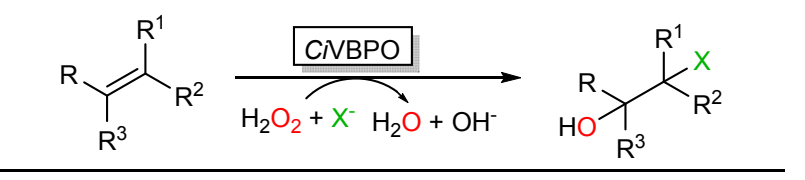<smiles>OC(CCl)c1ccccc1</smiles>

$77 \%^{a}$<smiles>OC(CBr)c1ccccc1</smiles>

$81 \%^{a}$<smiles>CC(O)(CBr)c1ccccc1</smiles><smiles>CC(Br)[C@H](O)c1ccccc1</smiles>
$68 \%^{a}$<smiles>C[C@H](Br)[C@H](O)c1ccccc1</smiles><smiles>O[C@H]1CCCC[C@@H]1Br</smiles>

$88 \%^{b}$
$78 \%^{a}$<smiles>CCCCCC(O)CBr</smiles><smiles>OC(CBr)Cc1ccccc1</smiles>
$51 \%^{b}$<smiles>O=[N+]([O-])c1ccc(C(O)CCl)cc1</smiles>
$60 \%^{b}$<smiles>O=[N+]([O-])c1ccc(C(O)CBr)cc1</smiles><smiles>CC(C)(C)CCCC(O)C(Br)CCCCCCCC(=O)O</smiles>

图 $5 \mathrm{VCPO}$ 催化 $\mathrm{C}=\mathrm{C}$ 的羟卤化反应的底物范围 Figure 5 Substrate scope of halohydroxylation of $\mathrm{C}=\mathrm{C}$ double bond catalyzed by VCPO

Reaction conditions: $[\mathrm{Ci} \mathrm{VCPO}]=100 \mathrm{nmol} \cdot \mathrm{L}^{-1}$; [substrate $]=40 \mathrm{mmol} \cdot$ $\mathrm{L}^{-1} ;[\mathrm{KX}, \mathrm{X}=\mathrm{Cl} 、 \mathrm{Br}]=160 \mathrm{mmol} \cdot \mathrm{L}^{-1} ;\left[\mathrm{H}_{2} \mathrm{O}_{2}\right]=170 \mathrm{mmol} \cdot \mathrm{L}^{-1}, 0.1 \mathrm{~mol} \cdot$ $\mathrm{L}^{-1}$ citrate buffer, $\mathrm{pH} 5.0, T=25{ }^{\circ} \mathrm{C}, t=20 \mathrm{~h} .{ }^{a}$ isolated yield. ${ }^{b}{ }^{1} \mathrm{H}$ NMR yield

环状卤代醚(图 6). 该反应的一个突出优势是，因底物小 分子羧酸具有良好的水溶性，使产物浓度可以达到 150 $\mathrm{mmol} \cdot \mathrm{L}^{-1}$ 以上，在量级制备方面具有良好的前景 ${ }^{[41-42]}$. 如图 7 所示, 多种取代的羧酸或醇可以发生环合反应, 且可以容易地分离提纯克级以上产物. 类似地, 当底物 从 4-戊炔酸出发时, 从红藻中分离得到的 VBPO 可催化 合成溴代不饱和呋喃酮 ${ }^{[43]}$. 虽然天然存在的溴代呋喃 酮的前体未知, 且不饱和 $\mathrm{C}=\mathrm{C}$ 存在于呋喃酮环中, 但 上述利用卤过氧化物酶合成的呋喃酮仍然能够有效抑 制根癌农杆菌. 因此，以上结果证明了卤过氧化物酶在 创制作物病菌抑制剂中极具开发潜力.

值得一提的是, $E$-因子 $[E$-factor $=m($ 废物 $) / m($ 产物 $)]$ 为衡量化学合成的绿色潜力提供一个量化方法 ${ }^{[44]}$. 通 过 $E$-因分子分析可以看出, 酶催化卤环合反应可以显 著降低废物排放, 减轻卤化反应可能带来的环境污染压 力 $^{[40-42]}$. 需要指出的是, 上述研究得到的产物都是外消 旋的, 即不具备立体(对映体)选择性. 为此, 考虑到内 酯和环醚产物在高分子合成、香精香料、有机合成的 


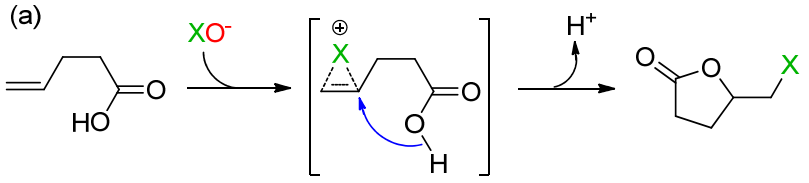

(1)

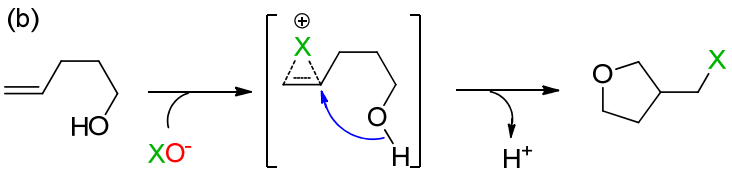

图 6 由酶生成的活性次卤酸催化分子内的卤环化反应机理 Figure 6 Mechanism of the intramolecular halo-cyclization reaction catalyzed by the reactive hypohalites

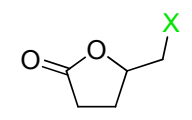

$X=\mathrm{Cl}, 99 \% ; 67 \%$ $\mathrm{X}=\mathrm{Br}, 99 \% ; 67 \%$

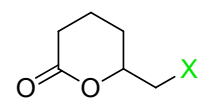

$X=\mathrm{Cl}, 99 \% ; 70 \%$ $\mathrm{X}=\mathrm{Br}, 99 \% ; 56 \%$

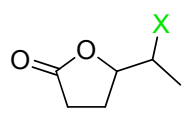

$X=\mathrm{Cl}, 99 \% ; 56 \%$ $\mathrm{X}=\mathrm{Br}, 99 \% ; 57 \%$<smiles>[X]CC1CCCCO1</smiles>

$X=\mathrm{Cl}, 99 \% ; 77 \%$ $\mathrm{X}=\mathrm{Br}, 99 \% ; 77 \%$

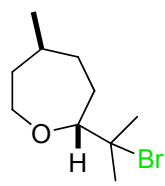

$52 \% ; 79 \%$
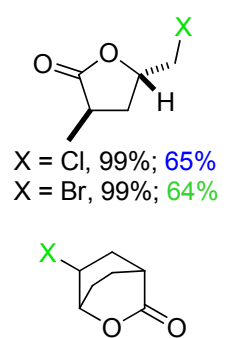

$X=\mathrm{Cl}, 99 \% ; 62 \%$ $\mathrm{X}=\mathrm{Br}, 99 \% ; 70 \%$

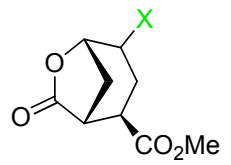

$$
\mathrm{X}=\mathrm{Cl}, 80 \% ; 87 \%
$$
$\mathrm{X}=\mathrm{Br}, 99 \% ; 79 \%$

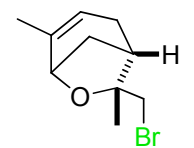

$67 \% ; 52 \%$

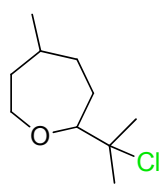

$95 \% ; 85 \%$

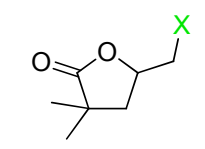

$\mathrm{X}=\mathrm{Cl}, 99 \% ; 68 \%$ $\mathrm{X}=\mathrm{Br}, 99 \% ; 72 \%$

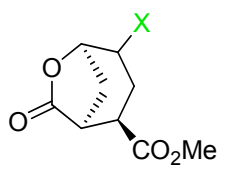

$\mathrm{X}=\mathrm{Cl}, 86 \% ; 82 \%$ $\mathrm{X}=\mathrm{Br}, 99 \% ; 80 \%$

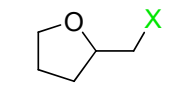

$\mathrm{X}=\mathrm{Cl}, 60 \% ; 78 \%$ $\mathrm{X}=\mathrm{Br}, 95 \% ; 81 \%$

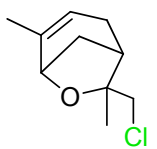

56\%; $72 \%$
图 $7 C i \mathrm{VCPO}$ 催化 $\gamma, \delta$-不饱和羧酸(醇)的环内酯化(环内醚化) 反应的转化率(蓝色)和选择性(绿色)

Figure $7 \mathrm{CiVCPO}$ catalyzed halo-cyclization reactions of $\gamma, \delta$-unsaturated carboxylic acids (alcohols) and the corresponding conversion (blue) and selectivity (green)

重要性, 未来的研究可以考虑利用高选择性的脂肪酶对 外消旋产物进行动力学拆分, 进而获得光学纯的终产 物.

\section{5 氧化脱羧反应}

上述提到的所有反应中, 酶生成的次卤酸盐起化学 计量试剂的作用, 并被引入到底物中, 那么, 活性次卤 酸盐能否以催化剂量参与化学反应? 研究表明, 由酶催 化生成的次卤酸盐也可以承担催化剂的角色. 其中一个
重要反应为 $\alpha$-氨基酸的氧化脱羧生成相应的 $\left(\mathrm{C}_{1}\right.$ 缩短的 $)$ 腈. Scott 等 ${ }^{[45-46]}$ 在 2012 和 2017 年开发了针对 VCPO 的 变体，可使活性次卤酸以催化剂量参与催化反应，从而 大幅度避免了化学计量的盐废物积累, 该研究对基于卤 化酶开发绿色化学工艺具有重要启发意义. 尽管该酶促 反应比传统铇催化的反应具有更高的 TON, 但是为了 获得更高经济效益, 底物的低负载量是亟待解决的瓶 颈. 2019 年底, Hollmann 等 ${ }^{[47]}$ 将谷氨酸的浓度提升到 $100 \mathrm{mmol} \cdot \mathrm{L}^{-1}$, 进行氧化脱羧制备 3-氧丙酸(图 8). 在生 物化工领域, 该反应有望成为将(废)氨基酸转化为腈基 砌块的实用方法. 但为了提高 $\mathrm{H}_{2} \mathrm{O}_{2}$ 的利用率及避免单 线态氧 $\left({ }^{1} \mathrm{O}_{2}\right)$ 的生成，必须控制 $\mathrm{H}_{2} \mathrm{O}_{2}$ 的投料速率，在一 定程度上增加了工艺的复杂性.
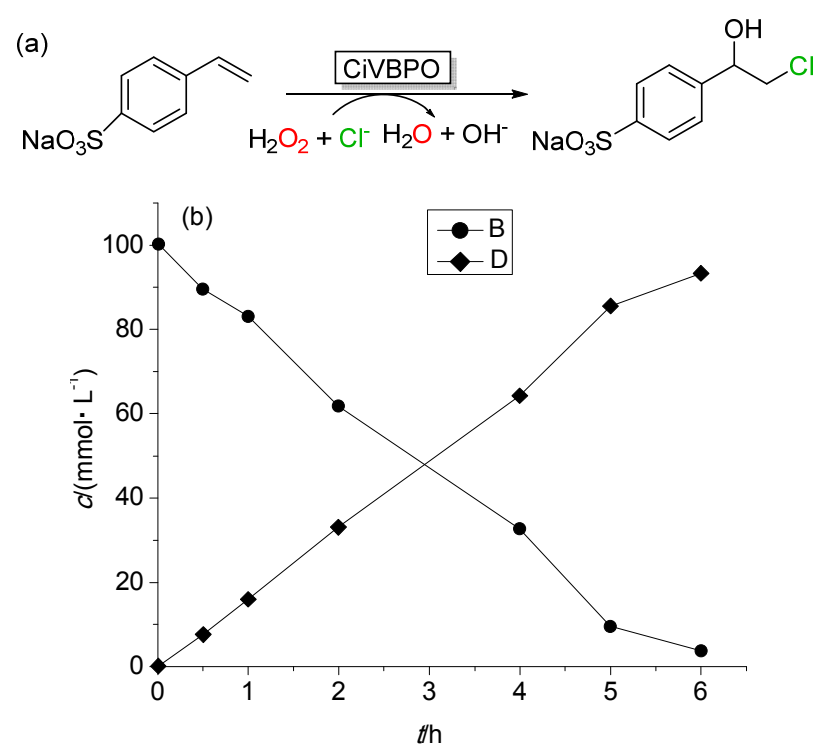

图 8 谷氨酸 $($ ) 的氧化脱羧制备 3 -氰丙酸 $($ )

Figure 8 Oxidative decarboxylation of glutamic acid (0) to synthesize 3-cyanopropionic acid $(\diamond)$

Reaction conditions: [glutamic acid] $=100 \mathrm{mmol} \bullet \mathrm{L}^{-1},[\mathrm{NaBr}]=5 \mathrm{mmol} \bullet$ $\mathrm{L}^{-1},[\mathrm{CiVCPO}]=50 \mathrm{nmol} \cdot \mathrm{L}^{-1}$, dose rate of $\mathrm{H}_{2} \mathrm{O}_{2} 39 \mathrm{mmol} \cdot \mathrm{L}^{-1} \cdot \mathrm{h}^{-1}$, citrate buffer $\left(20 \mathrm{mmol} \cdot \mathrm{L}^{-1}, \mathrm{pH} 5.6\right), T=22{ }^{\circ} \mathrm{C}$

\section{6 磺氧化反应}

光学纯亚砜是不对称合成中的重要合成子. 已有多 种氧化还原酶催化对映选择性的磺氧化反应(图 9). 其 中, AaeUPO 和 CPO 可对一系含硫底物进行氧化反应, 获得光学纯产物 ${ }^{[48-49]}$. 但因血红素型卤过化酶对 $\mathrm{H}_{2} \mathrm{O}_{2}$ 的高度敏感及底物的低溶解性, 使其在磺氧化反应中的 应用受到一定限制. 相较而言, VBPO 因与 $\mathrm{H}_{2} \mathrm{O}_{2}$ 共存时 的超级稳定性，而使其成对有机硫化物选择性磺氧化的 一个有利选择. 但因来源不同, VBPO 的催化性能差异 较大, 如来自褐紫菜 A. nodosum 的 VBPO 催化氧化苯硫 基甲烷时，得到 $R$ 型的产物( $92 \% e e)$, 其 TOF 可达 166 $\mathrm{s}^{-1}$. 而使用自红海藻 $C$. pilulifera 的 VBPO 则得到 $S$ 型 
产物(55\%ee), 且底物芳环上的上取代基对对映体选择 性有较大的影响.

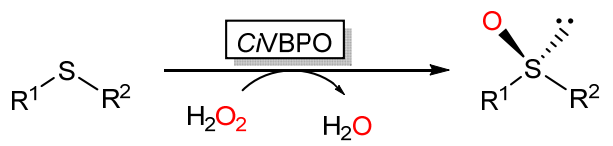

图 9 卤过氧化物酶催化磺氧化反应

Figure 9 Sulfoxidation reactions catalyzed by haloperoxidases

需要特别指出的是, 为了绕过次氯酸作为化学催化 剂参与磺氧化反应(导致外消旋产物), 卤化酶催化磺氧 化反应时无须添加卤素, 从而保证获得较高光学纯氧化 产物. 同时, $\mathrm{H}_{2} \mathrm{O}_{2}$ 须保持在较低浓度, 以阻止其与硫化 物的化学反应.

\section{7 其他反应}

除了较经典的卤化氧化反应外, Deska 等 ${ }^{[50]}$ 研究发 现, 与醇脱氢酶 $(\mathrm{ADH})$ 偶联时 $L f \mathrm{CPO}$ 具有的催化多功能 性可以催化呋喃氧化环扩张(Achmatowicz)反应, 得到 一系列具有光学纯度的呋喃醇(图 10), 但至今仍不明确 其催化反应的机理. 鉴于该反应在天然产物合成中的重 要性, 此方向的进一步研究值得期待.

\section{4 卤过氧化物酶的催化活性及立体选择性}

为了减轻使用化学计量的次卤酸盐而引起的选择 性和环境问题，常用的一个策略是开发卤素的原位氧 化. 迄今, 已开发了多种化学催化方法氧化相应的卤素 原位生成次卤酸盐的方法. 表 1 对比了化学催化剂与 $C i \mathrm{VCPO}$ 在催化溴代反应时的性能. 与 “经典” 化学催 化剂相比, $C i \mathrm{VCPO}$ 的负载量(间接地以 $\mathrm{TON}$ 表示)比化 学催化剂低 $1 \sim 6$ 个数量级. 这些比较数据证明了 $\mathrm{CiVC}-$ PO 优异的催化性能在开发清洁和高效的卤化反应方法 (a)

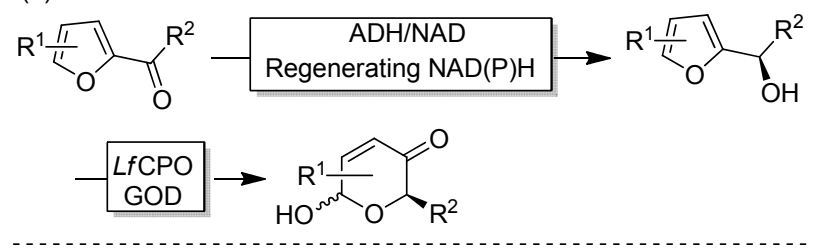<smiles>CC1O[C@H](O)C=CC1=O</smiles><smiles>C[C@H]1O[C@@H](O)C=CC1=O</smiles><smiles>CCC1O[C@H](O)C=CC1=O</smiles>

$77 \%, 99 \%$ ee $78 \%, 99 \%$ ee $70 \%, 99 \%$ ee<smiles>CCCC1O[C@@H](O)C=CC1=O</smiles><smiles>CC(C)C1O[C@H](O)C=CC1=O</smiles><smiles>CCOC(=O)CC1O[C@H](O)C=CC1=O</smiles><smiles>C=CCC1O[C@H](O)C=CC1=O</smiles>
$64 \%, 99 \%$ ee<smiles>C#CC1O[C@H](O)C=CC1=O</smiles>
$65 \%, 98 \%$ ee<smiles>O=C1C=C[C@@H](O)OC1CO</smiles>

$31 \%, 99 \%$ ee<smiles>CC1OC(C)(O)C=CC1=O</smiles>

图 $10 L f C P O$ 催化呋喃的氧化环扩张(Achmatowicz)反应(a)及 其底物范围(b)

Figure $10 \quad L f C P O-c a t a l y z e d$ oxidative ring-expansion (Achmatowicz) reactions of furans (a) and its substrate scope (b) Reaction conditions: [Glucose $]=50 \mathrm{mmol} \cdot \mathrm{L}^{-1},[$ Substrate $]=10 \mathrm{mmol} \cdot$ $\mathrm{L}^{-1}$, [Glucose oxidase $]=3.6 \mathrm{mmol} \cdot \mathrm{L}^{-1},[$ Substrate $]=10 \mathrm{mmol} \cdot \mathrm{L}^{-1}$, $[L f \mathrm{CPO}] 0.41 \mathrm{mmol} \cdot \mathrm{L}^{-1}, 100 \mathrm{mmol} \cdot \mathrm{L}^{-1}$ sodium citrate buffer $(\mathrm{pH} 5.5)$, $10 \%$ tert-butanol, r.t.

中具有重要价值.

但是，在以上两类卤过氧化物酶中，活性次卤酸盐 生成后从酶的孔道扩散出去，使接下来的催化反应脱离 了酶手性环境的支持 ${ }^{[12]}$. 因此，反应的选择性由底物的

表 $1 C i \mathrm{VCPO}$ 与传统化学催化剂在溴化反应和脱羧反应中的催化性能对比

Table 1 Comparison of the catalytic performance between $\mathrm{CiVCPO}$ and chemical counterparts in bromination and decarboxylation reactions

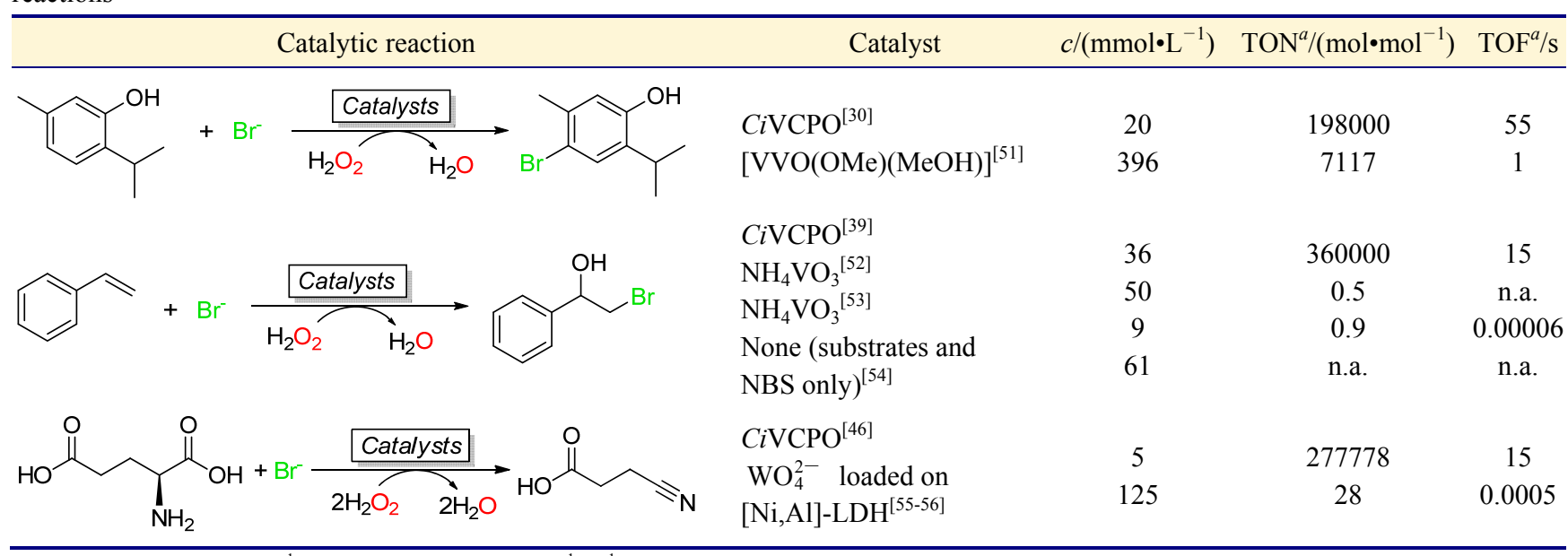

${ }^{a} \mathrm{TON}=\operatorname{mol}_{\text {product }} \cdot \mathrm{mol}_{\text {catalyst }}{ }^{-1}, \mathrm{TOF}=\operatorname{mol}_{\text {product }} \cdot \mathrm{mol}_{\text {catalyst }}{ }^{-1} \bullet \mathrm{s}^{-1}$. n.a. $=$ not available. Data from Höfler, G. T.; But, A.; Hollmann, F. Org. Biomol. Chem. 2019, 17, 9267. 
化学反应性(中间态 $\sigma$-复合物的离域度和稳定性)而非酶 控制. 但已有文献报道了个别例外：Moore 及其同 事 $^{[57-58]}$ 证明了来源于链霉菌的卤过氧化物酶具有高度 立体选择性, 然而, 该选择性卤化反应的分子机理尚不 清楚, 且报道的实例数据不足以完整阐述分子反应机 制. 另一方面, 我们已经看到, 于 2018 年获得诺贝尔化 学奖的人工酶定向进化技术 ${ }^{[59-60]}$, 经过 30 多年的发展, 已经成为改造及提升酶催化特性(如区域、立体、化学 选择性、催化活性、 $\mathrm{pH}$ 及热稳定性等)的有效技术方 法 ${ }^{[61]}$. 因此, 有理由期待未来将有更多的研究报道利用 定向进化技术提升卤过氧化物酶的立体催化选择性能.

\section{5 原位 $\mathrm{H}_{2} \mathrm{O}_{2}$ 生成与卤过氧化物酶的偶联}

血红素依赖型卤过氧化物酶, 如其他所有血红素依 赖型氧化酶一样, 对共底物 $\mathrm{H}_{2} \mathrm{O}_{2}$ 极其敏感. 例如, Holtmann 等 ${ }^{[34]}$ 探索了利用血红素依赖型 $L f C P O$ 来卤化 萜烯以提高其抗菌活性, 但最小化 $L f C P O$ 的血红素氧化 失活, 需使用气体扩散电极电化学还原 $\mathrm{O}_{2}$ 原位生成 $\mathrm{H}_{2} \mathrm{O}_{2}$. 钒依赖型卤过氧化物酶虽然对 $\mathrm{H}_{2} \mathrm{O}_{2}$ 具有良好的 耐受性, 但由于次卤酸盐和 $\mathrm{H}_{2} \mathrm{O}_{2}$ 之间的自发反应会产 生单线态氧 $\left({ }^{1} \mathrm{O}_{2}\right)$, 引起可能的副反应及降低 $\mathrm{H}_{2} \mathrm{O}_{2}$ 的利 用效率, 因此, 实际应用中仍需控制 $\mathrm{H}_{2} \mathrm{O}_{2}$ 的浓度. 为保 证卤化酶在催化循环过程中的稳定性、终产物的选择性 和浓度, 有必要开发原位 $\mathrm{H}_{2} \mathrm{O}_{2}$ 的生成技术, 从 $\mathrm{O}_{2}$ 还原 生成 $\mathrm{H}_{2} \mathrm{O}_{2}$ 是目前最具吸引力的方法. 目前, 已有基于电 化学法、光(电)催化法、酶催化法等一系可供过氧化物 酶偶联的技术方法 ${ }^{[34,41,62-68]}$. 值得一提的是, 作者开发 了一系列基于水或甲醇的光催化氧化技术(图 11) ${ }^{[64-65]}$, 以及在低电位下 $\mathrm{H}_{2} \mathrm{O}_{2}$ 原位生成方法 ${ }^{[68]}$, 分别用来促进 血红素依赖型过氧合酶和钒依赖型 $C i \mathrm{VCPO}$ 的氧化以 及卤化反应. 上述 $\mathrm{H}_{2} \mathrm{O}_{2}$ 原位生成方法为开发可控、高原 子经济性及清洁的卤过氧化物酶催化技术提供了可靠 的技术基础.

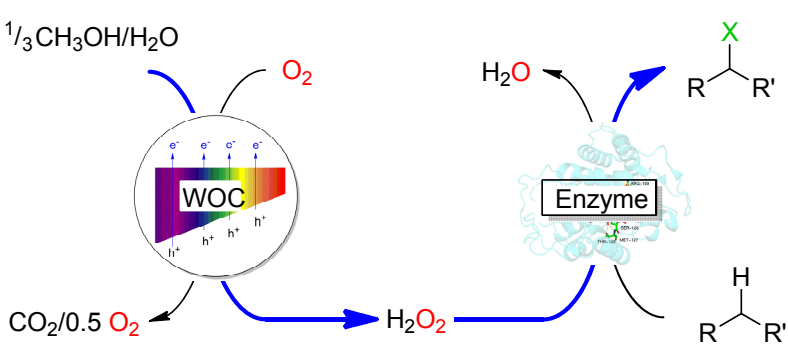

图 11 高原子经济性光催化氧化 $\mathrm{H}_{2} \mathrm{O}^{[64]}$ 和 $\mathrm{CH}_{3} \mathrm{OH}^{[65]}$ 原位生 成 $\mathrm{H}_{2} \mathrm{O}_{2}$ 级联过氧合酶催化 (蓝色箭头代表氧化反应中电子流 向)

Figure 11 High atom-efficient photocatalytic oxidation of $\mathrm{H}_{2} \mathrm{O}^{[64]}$ and $\mathrm{CH}_{3} \mathrm{OH}^{[65]}$ for the in situ generation of $\mathrm{H}_{2} \mathrm{O}_{2}$ and cascading with peroxygenases

另一方面, 由于非均相催化剂较低的催化效率, 其 与卤过氧化物酶联用时的低产物浓度依然是限制其实 用性的一个瓶颈. 鉴于此, 最近水溶性葱醌被用作光催 化剂, 对同时作为电子牺牲剂和溶液的小分子醇进行催 化氧化原位生成 $\mathrm{H}_{2} \mathrm{O}_{2}$ (图 12), 与 $C i \mathrm{VCPO}$ 联用时可显著 地提高反应速率及产物浓度, 在量级合成中可分离获得 克级的溴代百里香酚 ${ }^{[69]}$.

\section{6 结语}

开发清洁、高效、高选择性的卤化反应，对推动卤 化化学相关研究、绿色生物制造及卤化工业环境治理等 方面具有重要现实意义. $E$-因子分析表明，卤化酶与传 统化学催化剂相比, 在降低反应废物排放, 即绿色合成 方面, 具有突出优势. 其中, 卤过氧化物酶具有超高的 催化活性、稳定性及催化多功能性, 是绿色卤化反应中 一类非常具有实用潜力的催化剂. 为提高卤过氧化酶对 $\mathrm{H}_{2} \mathrm{O}_{2}$ 的耐受性及对 $\mathrm{H}_{2} \mathrm{O}_{2}$ 的利用效率，已有一系列原位 $\mathrm{H}_{2} \mathrm{O}_{2}$ 生成技术可与卤过氧化酶偶联应用.

当前, 在具体的卤化酶催化绿色卤化反应研究层 面, 尚存在两类主要挑战. 首先, 绝大多数卤过氧化物

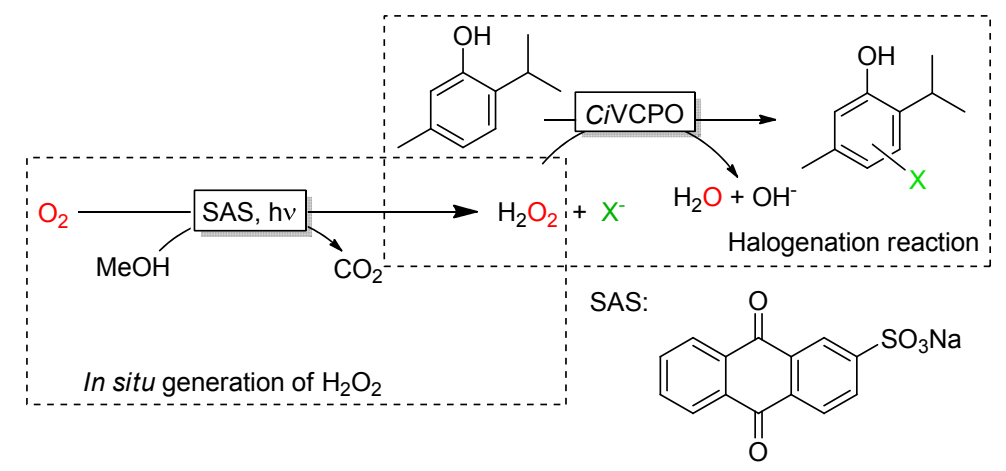

图 12 水溶性葱醌光催化氧化小分子醇生成 $\mathrm{H}_{2} \mathrm{O}_{2}$ 与 $\mathrm{CiVCPO}$ 偶联催化百里香酚的澳代反应

Figure 12 Water soluble anthraquinone catalyzed simple alcohol oxidation for the in situ generation of $\mathrm{H}_{2} \mathrm{O}_{2}$ and cascading with $\mathrm{Ci} \mathrm{VCPO}$ for the bromination of thymol 
酶依赖可扩散的次卤酸盐, 而使其失去生物催化的一个 重要特征: 由酶的三维结构支撑的立体选择性. 因此, 利用定向进化等分子生物学技术对卤过氧化物酶进行 分子改造, 将大力促进这类生物催化剂在选择性合成方 面的蓬勃发展. 其次, 生物催化在实践中普遍使用水作 为溶剂, 而有机底物的水溶性普遍较差. 为使卤过氧化 物酶成为规模化有机合成的实用催化剂, 可通过对反应 体系设计和优化, 以添加助溶剂, 或采用纯有机相与固 定化酶相结合的方式 ${ }^{[70]}$, 来实现大幅度提升底物的浓 度. 我们相信, 对上述挑战的解决, 必将促进卤过氧化 酶朝着更加实用的卤化反应催化剂方向发展, 助力绿色 有机合成及相关工业应用技术的开发.

\section{References}

[1] https: //njardarson. lab. arizona. edu/sites/njardarson. lab. arizona. edu/files/Top $\% 20200 \% 20$ Drugs $\% 20$ By $\% 20$ Retail $\% 20$ Sales $\% 20$ in $\% 202019$ V2. pdf

[2] Saikia, I.; Borah, A. J.; Phukan, P. Chem. Rev. 2016, 116, 6837.

[3] Alonso, F.; Beletskaya, I. P.; Yus, M. Chem. Rev. 2002, 102, 4009.

[4] Petrone, D. A.; Ye, J.; Lautens, M. Chem. Rev. 2016, 116, 8003.

[5] Gandeepan, P.; Müller, T.; Zell, D.; Cera, G.; Warratz, S.; Ackermann, L. Chem. Rev. 2019, 119, 2192.

[6] Teskey, C. J.; Lui, A. Y. W.; Greaney, M. F. Angew. Chem., Int. Ed. 2015, 54, 11677.

[7] Cheng, H.-G.; Chen, S.; Chen, R.; Zhou, Q. Angew. Chem., Int. Ed. 2019, $58,5832$.

[8] Gandeepan, P.; Koeller, J.; Korvorapun, K.; Mohr, J.; Ackermann, L. Angew. Chem., Int. Ed. 2019, 58, 9820.

[9] Sagadevan, A.; Greaney, M. F. Angew. Chem., Int. Ed. 2019, 58, 9826.

[10] Xu, H.; Shang, M.; Dai, H.-X.; Yu, J.-Q. Org. Lett. 2015, 17, 3830.

[11] Yu, J.-Q.; Shi, Z. Topics in Current Chemistry, Vol. 292, Springer GmbH, Germany, 2010.

[12] Latham, J.; Brandenburger, E.; Shepherd, S. A.; Menon, B. R. K.; Micklefield, J. Chem. Rev. 2018, 118, 232.

[13] Agarwal, V.; Miles, Z. D.; Winter, J. M.; Eustáquio, A. S.; El Gamal, A. A.; Moore, B. S. Chem. Rev. 2017, 117, 5619.

[14] Gribble, G. W. Progress in Chemistry of Organic Natural Products, Vol. 91, Springer, New York, 2010.

[15] Weichold, V.; Milbredt, D.; van Pée, K.-H. Angew. Chem. Int. Ed. 2016, 55, 6374 .

[16] Smith, D. R. M.; Grüschow, S.; Goss, R. J. M. Curr. Opin. Chem. Biol. 2013, 17, 276.

[17] Schallmey, A.; Schallmey, M. Appl. Microbiol. Biotechnol. 2016, 100, 7827.

[18] Leblanc, C.; Vilter, H.; Fournier, J. B.; Delage, L.; Potin, P.; Rebuffet, E.; Michel, G.; Solari, P. L.; Feiters, M. C.; Czjzek, M. Coord. Chem. Rev. 2015, 301-302, 134.

[19] Tufvesson, P.; Lima-Ramos, J.; Nordblad, M.; Woodley, J. M. Org. Process Res. Dev. 2011, 15, 266.

[20] Izumi, Y.; Xu, L.; di Tomaso, E.; Fukumura, D.; Jain, R. K. Nature 2002, 416, 279.

[21] Dong, C.-J.; Huang F.-L.; Deng, H.; Schaffrath C.; Spencer, J. B.; O'Hagan, D.; Naismith, J. H. Nature 2004, 427, 561.

[22] Hofrichter, M.; Ullrich, R. Appl. Microbiol. Biotechnol. 2006, 71, 276.

[23] Raugei, S.; Carloni, P. J. Phys. Chem. B 2006, 110, 3747.

[24] Noyori, R.; Aoki, M.; Sato, K. Chem. Commun. 2003, 1977.

[25] Xia, C.; Xia, Y.; Zhu, P.; Fan, L.; Wang, H. Science 2019, 366, 226

[26] Morris, D. R.; Hager, L. P. J. Biol. Chem. 1966, 241, 1763.

[27] Ullrich, R.; Nüske, J.; Scheibner, K.; Spantzel, J.; Hofrichter, M.
Appl. Environ. Microbiol. 2004, 70, 4575.

[28] Wever, R.; van der Horst, M. A. Dalton Trans. 2013, 42, 11778.

[29] Wever, R.; Krenn, B. E.; Renirie, R. In Methods in Enzymology, Vol. 605, Ed.: Moore, B. S., Academic Press, United States, 2018, p. 141.

[30] Fernandez-Fueyo, E.; van Wingerden, M.; Renirie, R.; Wever, R.; Ni, Y.; Holtmann, D.; Hollmann, F. ChemCatChem 2015, 7, 4035.

[31] Yu, Y.; Jin Y.; Wu, P.-C.; Zhang, W. Chin. J. Catal. 2007, 28, 915 (in Chinese). (于瑶，靳艳，吴佩春，张卫，催化学报, 2007, 28, 915.)

[32] Zhang, B.-M.; Cao, X.-P.; Xue, S.; Xiao, T.-H.; Zhang, W. Chin. J. Catal. 2010, 31, 1293 (in Chinese).

(章表明, 曹旭鹏, 薛松, 肖通虎, 张卫, 催化学报, 2010, 31, 1293.)

[33] Wischang, D.; Hartung, J. Tetrahedron 2012, 68, 9456.

[34] Getrey, L.; Krieg, T.; Hollmann, F.; Schrader, J.; Holtmann, D. Green Chem. 2014, 16, 1104.

[35] Kaur, R.; Darokar, M. P.; Chattopadhyay, S. K.; Krishna, V.; Ahmad, A. Med. Chem. Res. 2014, 23, 2212.

[36] Frank, A.; Seel, C. J.; Groll, M.; Gulder, T. ChemBioChem 2016, $17,2028$.

[37] Seel, C. J.; Králík, A.; Hacker, M.; Frank, A.; König, B.; Gulder, T. ChemCatChem 2018, 10, 3960.

[38] Carter-Franklin, J. N.; Butler, A. J. Am. Chem. Soc. 2004, 126, 15060.

[39] Dong, J. J.; Fernandez-Fueyo, E.; Li, J.; Guo, Z.; Renirie, R.; Wever, R.; Hollmann, F. Chem. Commun. 2017.

[40] Younes, S. H. H.; Tieves, F.; Lan, D.; Wang, Y.; Suess, P.; Brundiek, H.; Wever, R.; Hollmann, F. ChemSusChem 2020, 13, 1.

[41] Tieves, F.; Willot, S. J.; van Schie, M.; Rauch, M. C. R.; Younes, S. H. H.; Zhang, W.; Dong, J.; Gomez de Santos, P.; Robbins, J. M.; Bommarius, B.; Alcalde, M.; Bommarius, A. S.; Hollmann, F. Angew. Chem., Int. Ed. 2019, 58, 7873.

[42] Höfler, G. T.; But, A.; Younes, S. H. H.; Wever, R.; Paul, C. E.; Arends, I. W. C. E.; Hollmann, F. ACS Sustainable Chem. Eng. 2020, $8,2602$.

[43] Sandy, M.; Carter-Franklin, J. N.; Martin, J. D.; Butler, A. Chem. Commun. 2011, 47, 12086.

[44] Sheldon, R. A. Green Chem. 2017, 19, 18.

[45] But, A.; van Noord, A.; Poletto, F.; Sanders, J. P. M.; Franssen, M. C. R.; Scott, E. L. Mol. Catal. 2017, 443, 92.

[46] But, A.; Le Nôtre, J.; Scott, E. L.; Wever, R.; Sanders, J. P. M. ChemSusChem 2012, 5, 1199.

[47] Xu, X.; But, A.; Wever, R.; Hollmann, F. ChemCatChem 2020, 12, 2180.

[48] Li, Y.; Ma, Y.; Li, P.; Zhang, X.; Ribitsch, D.; Alcalde, M.; Hollmann, F.; Wang, Y. ChemPlusChem 2020, 85, 254.

[49] Bassanini, I.; Ferrandi, E. E.; Vanoni, M.; Ottolina, G.; Riva, S.; Crotti, M.; Brenna, E.; Monti, D. Eur. J. Org. Chem. 2017, 2017, 7186.

[50] Thiel, D.; Doknić, D.; Deska, J. Nat. Commun. 2014, 5, 5278.

[51] Maurya, M. R.; Uprety, B.; Avecilla, F.; Adão, P.; Costa Pessoa, J. Dalton Trans. 2015, 44, 17736.

[52] Andersson, M.; Conte, V.; Di Furia, F.; Moro, S. Tetrahedron Lett. 1995, 36, 2675.

[53] Conte, V.; Floris, B.; Galloni, P.; Silvagni, A. Pure Appl. Chem. 2005, 77, 1575 .

[54] Narender, M.; Reddy, M. S.; Nageswar, Y. V. D.; Rao, K. R. J. Mol. Catal. A 2006, 258, 10 .

[55] Claes, L.; Matthessen, R.; Rombouts, I.; Stassen, I.; De Baerdemaeker, T.; Depla, D.; Delcour, J. A.; Lagrain, B.; De Vos, D. E. ChemSusChem 2015, 8, 345 .

[56] Claes, L.; Verduyckt, J.; Stassen, I.; Lagrain, B.; De Vos, D. E. Chem. Commun. 2015, 51, 6528.

[57] Kaysser, L.; Bernhardt, P.; Nam, S.-J.; Loesgen, S.; Ruby, J. G.; Skewes-Cox, P.; Jensen, P. R.; Fenical, W.; Moore, B. S. J. Am. Chem. Soc. 2012, 134, 11988.

[58] Miles, Z. D.; Diethelm, S.; Pepper, H. P.; Huang, D. M.; George, J. 
H.; Moore, B. S. Nat. Chem. 2017, 9, 1235.

[59] Arnold, F. H. Angew. Chem., Int. Ed. 2018, 57, 4143.

[60] Fasan, R.; Jennifer Kan, S. B.; Zhao, H. ACS Catal. 2019, 9, 9775.

[61] Qu, G.; Zhu, T.; Jiang, Y.-Y.; Wu, B.; Sun, Z.-T. Chin. J. Biotechnol. 2019, 35, 1843 (in Chinese)

(曲戈, 朱粀, 蒋迎迎, 吴边, 孙周通, 生物工程学报, 2019, 35, 1843.)

[62] Freakley, S. J.; Kochius, S.; van Marwijk, J.; Fenner, C.; Lewis, R. J.; Baldenius, K.; Marais, S. S.; Opperman, D. J.; Harrison, S. T. L.; Alcalde, M.; Smit, M. S.; Hutchings, G. J. Nat. Commun. 2019, 10, 4178 .

[63] van Schie, M. M. C. H.; Zhang, W.; Tieves, F.; Choi, D. S.; Park, C. B.; Burek, B. O.; Bloh, J. Z.; Arends, I. W. C. E.; Paul, C. E.; Alcalde, M.; Hollmann, F. ACS Catal. 2019, 9, 7409

[64] Zhang, W.; Fernández-Fueyo, E.; Ni, Y.; van Schie, M.; Gacs, J.; Renirie, R.; Wever, R.; Mutti, F. G.; Rother, D.; Alcalde, M.; Hollmann, F. Nat. Catal. 2018, 1, 55.
[65] Zhang, W.; Burek, B. O.; Fernandez-Fueyo, E.; Alcalde, M.; Bloh, J. Z.; Hollmann, F. Angew. Chem., Int. Ed. 2017, 56, 15451.

[66] Ni, Y.; Fernández-Fueyo, E.; Baraibar, A. G.; Ullrich, R.; Hofrichter, M.; Yanase, H.; Alcalde, M.; van Berkel, W. J. H.; Hollmann, F. Angew. Chem. Int. Ed. 2016, 55, 798.

[67] Willot, S. J. P.; Fernández-Fueyo, E.; Tieves, F.; Pesic, M.; Alcalde, M.; Arends, I. W. C. E.; Park, C. B.; Hollmann, F. ACS Catal. 2019 9, 890 .

[68] Bormann, S.; van Schie, M. M. C. H.; De Almeida, T. P.; Zhang, W.; Stöckl, M.; Ulber, R.; Hollmann, F.; Holtmann, D. ChemSusChem 2019, 12, 4759 .

[69] Yuan, B.; Mahor, D.; Fei, Q.; Wever, R.; Alcalde, M.; Zhang, W.; Hollmann, F. ACS Catal. 2020, 10, 8277.

[70] Fernández-Fueyo, E.; Ni, Y.; Gomez Baraibar, A.; Alcalde, M.; van Langen, L. M.; Hollmann, F. J. Mol. Catal. B: Enzym. 2016, 134, 347.

(Fan, Y.) 